\title{
Ultrasound Imaging of the Sciatic Nerve Division in the Popliteal Fossa: A Volunteer Study
}

\author{
Eric P. Chiang*, Paul Dangerfield, Daniel Asay, Anita Cucchiaro, Jeffrey S. Berger
}

The George Washington University, School of Medicine \& Health Sciences, Washington DC, USA.

Email: *eric.p.chiang@gmail.com

Received May $8^{\text {th }}, 2013$; revised June $15^{\text {th }}, 2013$; accepted July $6^{\text {th }}, 2013$

Copyright (C) 2013 Eric Chiang et al. This is an open access article distributed under the Creative Commons Attribution License, which permits unrestricted use, distribution, and reproduction in any medium, provided the original work is properly cited.

\begin{abstract}
The Background and Objectives: A sciatic nerve block at the level of the popliteal fossa is frequently administered for post-operative analgesia for surgery below the knee. While ultrasound continues to gain popularity as the technique of choice for guiding needle positioning during peripheral nerve blocks, practitioners can begin to utilize ultrasound to look for patterns of anatomical significance. Recognizing anatomical variations among different demographic populations can help practitioners improve in performing nerve blocks. We aim to determine if predictable variability exists in sciatic nerve bifurcation location and depth at the level of the popliteal fossa. Methods: After IRB approval, eligible subjects were screened for ASA I or II status and demographic data was collected. Fifty subjects were enrolled. The SonoSite MicroMaxx ${ }^{\circledR}$ with 38-mm broadband linear array, 13 - $6 \mathrm{MHz}$ probe with color Doppler and image capturing capabilities was used for ultrasound measurements. With subject lying prone, the location of the sciatic nerve in relation to the popliteal crease and skin-to-nerve distance were assessed via ultrasound. Two independent investigators confirmed nerve location for measurements. Analyses were performed with SAS version 9.1 using Pearson Correlation Coefficients and regression analysis. Results: Gender stratification revealed that, while males were both taller and heavier, skin-nerve measurements for depth were consistently deeper in females ( $\mathrm{p}$-value 0.02 ). Independent of the right or left leg, male gender and increased height decreases the skin-nerve distance, while increased weight increases the distance. There was no correlation between patient characteristics and crease-nerve distance. In some subjects, variability of crease-nerve distance even existed between their right and left leg. Conclusion: We show that significant variability exists for actual sciatic nerve bifurcation location, or target injection site, with consistently deeper skin depth values for female patients when compared to male patients, accounting for height and weight. These findings suggest visualization techniques such as ultrasound may lead to better localization of ideal injection sites.
\end{abstract}

Keywords: Ultrasonography; Nerve Block Methods; Peripheral Nerve Block; Popliteal Fossa; Sciatic Nerve; Analgesia Methods

\section{Introduction}

A distal sciatic nerve block in the popliteal fossa is frequently indicated for anesthesia and post-operative analgesia for lower extremity surgery below the knee. The sciatic nerve is formed from the L4-S3 spinal segments and divides into the tibial nerve (TN) and common peroneal nerve $(\mathrm{CPN})$. The ideal injection of local anesthetic should be positioned next to the main trunk of the sciatic nerve before its bifurcation to avoid an incomplete block $[1,2]$. The textbook posterior approach to the distal sciatic nerve is performed approximately inserting a needle $7-8 \mathrm{~cm}$ proximal to the crease formed

${ }^{*}$ Corresponding author. in the popliteal fossa when the knee is flexed 90 degrees. The technique does not factor in patient characteristics and anatomical variations of the nerve [3]. An anatomical variation in the division of the sciatic nerve is seen as a possible cause for incomplete blocks. Based on cadaveric specimens, the bifurcation of the sciatic nerve occurs less than $8 \mathrm{~cm}$ from the popliteal crease in only $75 \%$ of the legs investigated, which means the injection site could be distal to the bifurcation in $25 \%$ of patients when using the classic approach [1]. Anatomic variations were further analyzed with handheld ultrasound where the division was visualized in $72 \%$ of volunteers [4]. The purpose of the current study is to use ultrasound imaging to further analyze and determine if predictable variability exists in 
the location of bifurcation and depth of the sciatic nerve in the popliteal fossa.

\section{Methods}

After institutional review board approval (The George Washington University, Washington, DC) and written informed consent, 50 healthy volunteers participated in this study. The subjects provided weight and height measurements and answered screening questions to ensure ASA I or II status. Subjects were then asked to lie prone with bilateral lower extremities exposed. The SonoSite MicroMaxx ${ }^{\circledR}$ with $38 \mathrm{~mm}$ broadband linear array, 13 - 6 $\mathrm{MHz}$ probe with color Doppler and image capturing capabilities was used for sonography. Distance from the popliteal crease to the sciatic nerve was measured on the skin with ruler and marking pen.

The depth of the sciatic nerve from the skin was measured by the ultrasound point-to-point caliper ruler and was documented as skin-to-nerve distance. Images of the nerve and measurements were captured via the ultrasound machine. Image quality was based on the ability of two independent, expert observers (JB, PD), one performing ultrasound scanning and one observing, to identify the sciatic nerve with degree of confidence noted as follows: "Good," for two investigator certainty; "Fair," for one investigator certainty; or "Poor," if neither investigator could be certain of sciatic nerve bifurcation location.

Analyses were done with SAS version 9.1. A significance level of $<0.05$ was used, and all tests were twosided. Only data from ultrasound imaging rated as "good" was included for analysis.

\section{Results}

Fifty ASA I and II classification subjects were enrolled. According to predetermined criteria, the sciatic nerve division in the popliteal fossa was visualized in 47 of 50 (94\%) volunteers. A total of 43 subjects were used for the final data analysis $(4 \%$, or $8 \%$, dropped due to missing data; $3 \%$, or $6 \%$, were dropped due to fair or poor ultrasound identification of nerve). Subjects were ages 19 - 59 (mean 34.9 years old), 18 female and 25 male, height (154.9 - $193.0 \mathrm{~cm}$ or 61"- 76") (mean $173.4 \mathrm{~cm}\left(68^{\prime \prime}\right)$ ), and had no history of lower extremity trauma or congenital abnormalities (Table 1).

For the skin-nerve distance (depth of sciatic nerve at

Table 1. Patient characteristics.

\begin{tabular}{cc}
\hline Age (years) & $34.9+8.7$ \\
\hline Gender (Male/Female) & $25 / 18$ \\
Weight $(\mathrm{kg})$ & $72.4+14.1$ \\
Height $(\mathrm{cm})$ & $173.4+10.5$ \\
BMI $\left(\mathrm{kg} / \mathrm{m}^{2}\right)$ & $23.9+3.3$ \\
\hline
\end{tabular}

level of bifurcation), the mean for female subjects is 1.70 $\mathrm{cm}$ with a $95 \%$ confidence interval from $1.52-1.88$. The mean for male subjects is $1.46 \mathrm{~cm}$ with a $95 \%$ confidence interval of 1.34 - 1.58 (Figure 1). The t-test comparing the population means of the two groups yields a p-value of 0.02 , indicating that females have greater mean skin-nerve distances than males. Comparing the skin-nerve distance between the right and left leg of the same subject reveals the absolute difference between legs is $0.29 \mathrm{~cm}(95 \% \mathrm{CI}-$ $0.25,0.83$ ).

To predict the skin-nerve distance, a mathematical model can be constructed as follows: SKIN $=12.224-$ $0.1216 *$ WEIGHT $+0.0003 *$ WEIGHT $2-13.296 *$ GENDER + $0.1527 *$ GENDER * WEIGHT $-0.0004 *$ GENDER * WEIGHT 2, where GENDER $=1$ for female, and GENDER $=0$ for male.

The F-test for the usefulness of the model has a p-value of 0.0004 , which suggests that the model is useful in predicting the skin-nerve distance. The adjusted-R2 for the model is 0.201 , which means $20.1 \%$ of the variability in the skin-nerve distance can be explained by the model. In addition, the t-tests for the parameter coefficients in the model all have p-values less than 0.01 (Table 2), which shows significant linear relationship between skin-nerve distance and every covariate in the model.

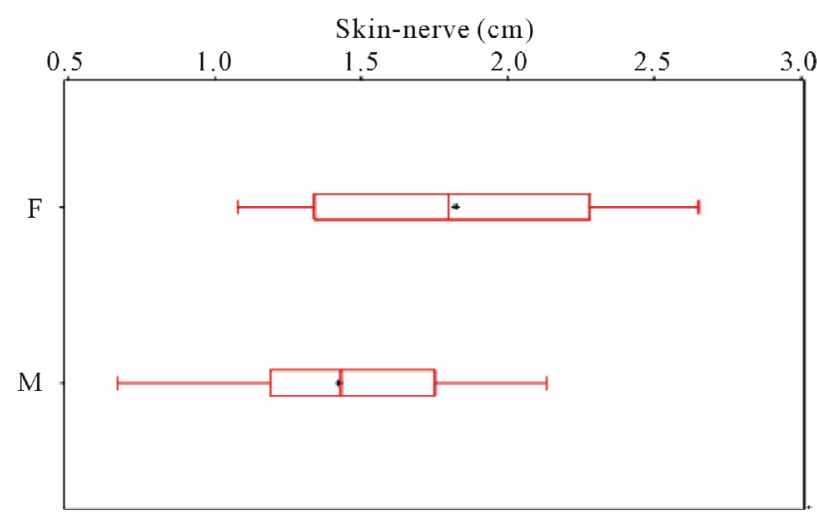

Figure 1. Gender stratification for skin-nerve distance.

Table 2. Parameter estimates of model to predict the skinnerve distance.

\begin{tabular}{cccccc}
\hline \multicolumn{5}{c}{ Parameter Estimates } \\
Variable & DF & Estimate & Error & t Value & $\operatorname{Pr}>|\mathrm{t}|$ \\
Intercept & 1 & 12.22429 & 3.74387 & 3.27 & 0.0016 \\
Weight & 1 & -0.12165 & 0.04261 & -2.85 & 0.0055 \\
Weight2 & 1 & 0.00033985 & 0.00012060 & 2.82 & 0.0061 \\
Gender & 1 & -13.29633 & 4.14905 & -3.20 & 0.0020 \\
Gender*Weight & 1 & 0.15274 & 0.04908 & 3.11 & 0.0026 \\
Gender*Weight2 & 1 & -0.00041441 & 0.00014466 & -2.86 & 0.0054 \\
\hline
\end{tabular}


For the crease-nerve distance, the mean for female subjects is $5.77 \mathrm{~cm}(95 \%$ CI $5.35,6.20)$, and the mean for male subjects is $6.02 \mathrm{~cm}(95 \%$ CI 5.48, 6.55). The t-test comparing the population means of the two groups does not distinguish a difference between genders. There were 18 of $43(42 \%)$ subjects that had crease-nerve distance greater than $7 \mathrm{~cm}$ and 10 of $43(21 \%)$ subjects had creasenerve distance of $8 \mathrm{~cm}$ or more. Comparing the creasenerve distance between the right and left leg of the same subject, the absolute difference for crease-nerve distance is $1.24 \mathrm{~cm}(95 \% \mathrm{CI}-1.09,3.56)$. However, 12 of 43 (28\%) subjects had an opposite leg difference in crease-nerve distance greater than $2 \mathrm{~cm}$.

We also tried different models to predict the creasenerve distance from the covariates and their transformations. However, we cannot find a significantly useful model to predict the crease-nerve distance, and none of the models we tried had a p-value less than 0.05 for the F-test of the model usefulness.

\section{Discussion}

Nerve localization techniques for regional anesthesia have evolved from paresthesia or peripheral nerve stimulation using anatomical landmarks to the more recent use of ultrasound guidance. There is insufficient evidence when measuring acute pain outcomes, and the question remains whether ultrasound techniques are superior to traditional landmark techniques incorporating nerve stimulation. [5].

The current literature overall does favor ultrasound guidance in technical block-related outcomes such as performance time, block onset, local anesthetic dose, and sensory block quality [6]. Ultrasound guidance allows not only for real-time, direct visualization of nerve structures, needle advancement, and local anesthetic spread, but also for detection of anatomic variations that are known to exist when compared to the anatomic dissections of cadavers illustrated in textbooks $[7,8]$.

Vloka et al. demonstrated that anatomic variations exist where the sciatic nerve divides into the tibial nerve and the common peroneal nerve after dissection of the popliteal fossa in 15 adult cadavers. The distance above the popliteal fossa crease to the bifurcation varied from 0 to 115 $\mathrm{mm}$, and with their anatomic model, a needle inserted at $50 \mathrm{~mm}, 70 \mathrm{~mm}$, or $80 \mathrm{~mm}$ above the popliteal fossa crease would only be proximal to the division of the sciatic nerve in $46 \%, 57 \%$, and $75 \%$, respectively [1]. This is clinically relevant because the sciatic nerve block in the popliteal fossa under nerve stimulation or paresthesia is often performed at $70 \mathrm{~mm}$ or $80 \mathrm{~mm}$ above the popliteal fossa crease and local anesthetic injected after response is obtained in the distribution of only one division of the nerve. Although there is a common epineural sheath that may carry the local anesthetic to the other division, an extraepineural injection at a location distal to the bifur- cation is the likely cause of the variable success rate of popliteal sciatic nerve blocks under traditional landmark with nerve stimulation techniques [2].

There have been attempts to modify the traditional landmark approach in order to capture the sciatic nerve before its bifurcation in more patients. Nader et al. compared nerve stimulator-guided popliteal sciatic nerve block with a classic approach performed $7-8 \mathrm{~cm}$ to a modified intertendinous approach performed $12-14 \mathrm{~cm}$ above the popliteal crease. They found complete block achieved in 39 of $54(79.9 \%)$ patients in the classic posterior group compared to 44 of $55(81.5 \%)$ patients in the modified intertendinous group [3].

Schwemmer et al. further characterized the anatomic variations of the sciatic nerve division using handheld ultrasound and attempted to correlate the ultrasound findings with patient's characteristics. They visualized the sciatic nerve division in 53 of $74(72 \%)$ volunteers and concluded that a relationship existed between the width of the popliteal crease and the depth of the nerve division, but there was no significant correlation in the crease-nerve distance and the patient's characteristics [4].

Our study adds to these findings as we analyzed the sciatic nerve division in 43 volunteers. After gender stratification, we found that while males were both taller and heavier, depth of the nerve division was consistently deeper in females. Specifically, after regression analysis, females of the same age, weight, and height have an expected nerve division that is $0.40 \mathrm{~cm}$ deeper than males. Adipose tissue appears to be a more significant regulator than skeletal tissue with regard to skin-nerve depth [9]. This is reflected in our data, where intuitively, greater weight increased skin-nerve depth. It is also reflected in the results of men and women matched for height and weight showing disparities in skin-nerve depth, with women consistently having a deeper skin-nerve depth. This is likely due to two factors: 1) women tend to accumulate adipose tissue preferentially in lower body, and 2) the adipose tissue accumulated is subcutaneous in nature. Adipose tissue distribution in humans is correlated to sex hormone levels and enzyme behavior differences in men and women. That women tend to accumulate more adipose tissue in the lower body is thought to be due to women's increased post-prandial lipoprotein lipase activity and decreased responsiveness to lipolysis in these anatomic areas $[10,11]$.

We were also able to normalize for patient characteristics by comparing the right and left legs in each volunteer. While the absolute difference for crease-nerve distance was not statistically significant, there were 12 of $43(28 \%)$ subjects that had variations in bifurcation site of $2 \mathrm{~cm}$ or more. A difference of $2 \mathrm{~cm}$ could be clinically relevant when performing the block in the case of an extraepineural injection. Injections of $30 \mathrm{ml}$ of local anesthetic 
into the epineural sheath would result in a proximal spread of $17.2 \mathrm{~cm}$ and would likely block both the tibial and common peroneal nerves regardless of bifurcation [2]. For an extra-epineural injection, a $30 \mathrm{ml}$ spherical volume would only have a radius of $1.93 \mathrm{~cm}$, which means if the injection is distal to the bifurcation, incomplete block is possible.

Numerous recent studies have compared ultrasound guidance versus nerve stimulation guidance alone for the sciatic nerve block at the popliteal fossa [12-15]. Gelfand et al. performed a meta-analysis to determine if ultrasound guidance improves success rate of a variety of nerve blocks. Ultrasound guidance was associated with the most significant increase in the success rate for sciatic popliteal nerve block $(\mathrm{RR}=1.22$ [95\% CI: 1.08 to $1.39, \mathrm{p}=0.002])$ [16]. This may be due to a greater degree of anatomic variation in nerve location for this block compared to other commonly performed blocks.

Limitations in this study were the inherent variability in operator mechanics for finding the sciatic nerve with ultrasound and the small sample size of the study. While using a single operator for nerve identification likely increased the internal validity of the study, external validity is less certain. Adding a second expert reviewer mitigated the effects of potential operator bias in measurements. It is also possible that, were more subjects enrolled in the study, further correlations and mathematical modeling predictions would have been evident. A larger study that utilizes more operators and subjects would be useful to expand upon the data presented.

\section{Conclusion}

The challenge of predicting sciatic nerve injection site is exemplified by the variation in bifurcation location that exists between the right and left leg among individual subjects. Our study has also shown that patient characteristics such as weight and gender can help predict the depth of the sciatic nerve from the skin using a mathematical model. Future studies should confirm these findings and investigate other characteristics on a larger scale. Ultimately, mathematical modeling may be incorporated into ultra-sound-guidance software to improve operator success with peripheral nerve blocks.

\section{Acknowledgements}

The authors gratefully acknowledge the assistance of Ruihua $\mathrm{Xu}$ and Linglu Wang for providing statistical analysis and Florence Ling for help with preparation of the manuscript.

\section{REFERENCES}

[1] J. D. Vloka, A. Hadzic, E. April and D. M. Thys, "The
Division of the Sciatic Nerve in the Popliteal Fossa: Anatomical Implications for Popliteal Nerve Blockade," Anesthesia \& Analgesia, Vol. 92, No. 1, 2001, pp. 215-217. doi:10.1097/00000539-200101000-00041

[2] J. D. Vloka, A. Hadzic, J. B. Lesser, E. Kitain, H. Geatz, E. W. April and D. M. Thys, "A Common Epineural Sheath for the Nerves in the Popliteal Fossa and Its Possible Implications for Sciatic Nerve Block," Anesthesia \& Analgesia, Vol. 84, No. 2, 1997, pp. 387-390.

[3] A. Nader, M. C. Kendall, K. D. Candido, H. Benzon and R. J. McCarthy, "A Randomized Comparison of a Modified Intertendinous and Classic Posterior Approach to Popliteal Sciatic Nerve Block," Anesthesia \& Analgesia, Vol. 108, No. 1, 2009, pp. 359-363. doi:10.1213/ane.0b013e31818c9452

[4] U. Schwemmer, C. K. Markus, C. A. Greim, J. Brederlau, M. Kredel and N. Roewer, "Sonographic Imaging of the Sciatic Nerve Division in the Popliteal Fossa," Ultraschall in der Medizin, Vol. 26, No. 6, 2005, pp. 496-500. doi:10.1055/s-2005-858531

[5] S. Choi and R. Brull, "Is Ultrasound Guidance Advantageous for Interventional Pain Management? A Review of Acute Pain Outcomes," Anesthesia \& Analgesia, Vol. 113, No. 3, 2011, pp. 596-604.

[6] J. Antonakakis and B. Sites, "Equivalence is Victory," Anesthesia \& Analgesia, Vol. 113, No. 3, 2011, pp. 439440. doi:10.1213/ANE.0b013e3182285e16

[7] A. T. Gray, "Ultrasound-Guided Regional Anesthesia: Current State of the Art," Anesthesiology, Vol. 104, No. 2, 2006, pp. 368-373. doi:10.1097/00000542-200602000-00024

[8] G. Retzl, S. Kapral, M. Greher and W. Mauritz, "Ultrasonographic Findings of the Axillary Part of the Brachial Plexus," Anesthesia \& Analgesia, Vol. 92, No. 5, 2001, pp. 1271-1275. doi:10.1097/00000539-200105000-00037

[9] C. D. Franco, N. Choksi, A. Rahman, G. Voronov and M. H. Almachnouk, "A Subgluteal Approach to the Sciatic Nerive in Adults at $10 \mathrm{~cm}$ From the Midline," Regional Anesthesia and Pain Medicine, Vol. 31, No. 3, 2006, pp. 215-220.

[10] S. B. Votruba and M. D. Jensen, "Sex-Specific Differences in Leg Fat Uptake are Revealed with a High-Fat Meal," Endocrinology and Metabolism: American Journal of Physiology, Vol. 291, No. 5, 2006, pp. E1115E1123. doi:10.1152/ajpendo.00196.2006

[11] C. M. Williams, "Lipid Metabolism in Women," Proceedings of the Nutrition Society, Vol. 63, No. 1, 2004, pp. 13-160. doi:10.1079/PNS2003314

[12] E. Dufour, P. Quennesson, A. L. Van Robais, F. Ledon, P. A. Laloe, N. Liu and M. Fischler, "Combined Ultrasound an Neurostimulation Guidance for Popliteal Sciatic Nerve Block: A Prospective, Randomized Comparison with Neurostimulation Alone," Anesthesia \& Analgesia, Vol. 106, No. 5, 2008, pp. 1553-1558. doi:10.1213/ane.0b013e3181684b42

[13] A. Perlas, R. Brull, V. W. Chan, C. J. McCartney, A. Nuica and S. Abbas, "Ultrasound Guidance Improves Success of Sciatic Nerve Block at the Popliteal Fossa," 
Regional Anesthesia and Pain Medicine, Vol. 33, No. 3, 2008, pp. 259-265.

[14] G. J. van Geffen, E. van den Broek, G. J. Braak, et al., "A Prospective Randomised Controlled Trial of Ultrasound Guided Versus Nerve Stimulation Guided Distal Sciatic Nerve Block at the Popliteal Fossa," Anaesthesia and Intensive Care, Vol. 37, No. 1, 2009, pp. 32-37.

[15] T. F. Bendtsen, T. D. Nielsen, C. V. Rohde, K. Kibak and F. Linde, "Ultrasound Guidance Improves a Continuous Popliteal Sciatic Nerve Block When Compared With Ner- ve Stimulation," Regional Anesthesia and Pain Medicine, Vol. 36, No. 2, 2011, pp. 181-184. doi:10.1097/AAP.0b013e31820d421f

[16] H. J. Gelfand, J. P. Ouanes, M. R. Lesley, P. S. Ko, J. D. Murphy, et al., "Analgesic Efficacy of Ultrasound-Guided Regional Anesthesia: A Meta-Analysis," Journal of Clinical Anesthesia, Vol. 23, No. 2, 2011, pp. 90-96. doi:10.1016/i.jclinane.2010.12.005 